\title{
LA ESTRATEGIA DEL BIG DATA COMO FACTOR CLAVE DE COMPETITIVIDAD EN LAS EMPRESAS
}

\section{THE STRATEGY OF BIG DATA AS A KEY FACTOR OF COMPETITIVENESS IN COMPANIES}

\author{
Dra. Yolanda González Castro*, MSc. Marta Milena Peñaranda Peñaranda** \\ MSc. Omaira Manzano Durán **** \\ "UNAD, Escuela de Ciencias Administrativas, contables económicas y de negocios. Grupo de \\ investigación GRINDES. UNAD Pamplona (N. de S), Colombia \\ 3174323093,yolanda.gonzalez@unad.edu.co \\ ** UFPSO, Facultad de Ciencias Administrativas y Económicas, Grupo de Investigación GIDSE \\ UFPS Ocaña (N. de S), Colombia. \\ 3213522287, mmpenarandap@ufpo.edu.co \\ **** UNAD, Escuela de Ciencias Administrativas, contables económicas y de negocios. Grupo de \\ investigación GRINDES. UNAD Ocaña (N. de S), Colombia \\ 3167442954,omaira.manzano@unad.edu.co
}

\begin{abstract}
Resumen: El uso masificado de las tecnologías y las redes sociales han permitido un aumento en los datos como fuente de información y conocimiento. Sin embargo, cada vez es más difícil el manejo de grandes cantidades de información en las organizaciones, especialmente cuando se quieren hacer modelación de variables para la toma de decisiones. Es por ello que en el mundo aparece el Big Data como herramienta para la selección, búsqueda y tratamiento de los datos existentes. Ahora bien, los avances que una herramienta tecnológica aporta en un sector específico se ven representado en las bases de datos científicas y tecnológicas. Es por ello que para encontrar una relación entre el Big Data y los negocios se realizó una investigación cuantitativa mediante una bibliometría, un estado de la ciencia y un estado de la técnica. Encontrándose 3889 documentos que integran la temática de Big Data con los negocios y los avances de Colombia con el resto del mundo.
\end{abstract}

Palabras clave: Big Data, Negocios, organizaciones, tecnología, toma de decisiones

\begin{abstract}
The widespread uses of technologies and social networks have allowed an increase in data as a source of information and knowledge. However, it is increasingly difficult to manage large amounts of information in organizations, especially when they want to model variables for decision making. That is why in the world Big Data appears as a tool for the selection, search and treatment of existing data. Now the advances that a technological tool contributes in a specific sector are represented in the scientific and technological databases. That is why to find a relationship between Big Data and business, quantitative research was carried out through bibliometrics, a state of science and a state of the art. Finding 3889 documents that integrate the theme of Big Data with business and the progress of Colombia with the rest of the world.
\end{abstract}

Keywords: Big Data, Business, Organizations, Technology, Decision Making 


\section{INTRODUCCIÓN}

Hoy en día las empresas presentan una gran demanda de tecnologías para solucionar muchos de sus problemas o situaciones de negocios (Parra \& Herrera, 2013). Incluso, algunas organizaciones tanto públicas como privadas requieren una integración de tecnologías para un manejo transversal de su operación tradicional (Bonfante \& Castillo, 2013). En muchos de los casos la implementación de diferentes tipos de tecnologías en los negocios permite entre otras ventajas mejorar los procesos de funcionamiento y calidad de la información, así como también poder realizar consultas permanentes en las bases, donde se alojan los datos (Sandoval, Mosquera \& González, 2011).

Hoy en día las empresas presentan una gran demanda de tecnologías para solucionar muchos de sus problemas o situaciones de negocios (Parra \& Herrera, 2013). Incluso, algunas organizaciones tanto públicas como privadas requieren una integración de tecnologías para un manejo transversal de su operación tradicional (Bonfante \& Castillo, 2013). En muchos de los casos la implementación de diferentes tipos de tecnologías en los negocios permite entre otras ventajas mejorar los procesos de funcionamiento y calidad de la información, así como también poder realizar consultas permanentes en las bases, donde se alojan los datos (Sandoval, Mosquera \& González, 2011). De igual forma las tecnologías han incursionado en diferentes campos tanto empresariales como sociales entre ellos los relacionados con las áreas de salud y la educación (Mesa \& Lombana, 2013) y algunas aplicaciones para población especial (Guzmán-Luna, Torres y Álvarez, 2014).

Vivimos un vertiginoso avance tecnológico sin precedentes, trayendo como consecuencia un acelerado desarrollo del conocimiento científico no antes visto en la historia de la humanidad. Esta creciente era digital ha dado surgimiento al almacenamiento de grandes cantidades de información estructurada y no estructurada. La gran cantidad de información disponible ha hecho que los estudiosos se pregunten qué hacer con tantos datos y cómo pueden servir eficientemente a los diferentes interesados.

En este sentido la Fundación Innovación Bankinter expresa que la ingente cantidad de datos disponibles ha hecho necesario el desarrollo de herramientas que los analicen y procesen para identificar lo relevante. Esta velocidad en la generación de datos ha propiciado el desarrollo de una nueva ciencia: el Big Data, que surge como una necesidad de entender y poder aprovechar la información disponible. Es así como Malvicino, F. y Yoguel, G., (2014), conciben la Big Data como un fenómeno global, que puede llegar a tener un impacto económico, beneficiando a los sectores público y privado, aumentando la productividad, la competitividad, y la calidad de vida.

Si bien podría considerarse lo definido por Russom, (2012), quien indica que "Big Data consiste en consolidar toda la información de una organización y ponerla al servicio del negocio", lo que nos llevaría a inferir que la Big Data ha cobrado cada vez más fuerza e interés para mejorar la toma de decisiones en los negocios, siendo esta la primera y más importante habilidad administrativa de un gerente (Drucker, 2013). Pero la gran pregunta de los empresarios según Tang Tong M, (2015), radica en cómo mantenerse competitivos y crecer en un mercado más globalizado, donde el cambio, principalmente liderado por la tecnología, rompe los paradigmas, apareciendo nuevos consumidores, nuevos productos, nuevos competidores y nuevas formas de hacer negocio, donde la Big Data se convierte en una herramienta para que los gerentes puedan medir y saber precisamente de una forma radical lo que está sucediendo en sus negocios y traducir directamente ese conocimiento en una toma de decisiones mejorada y en un rendimiento superior. (Goyzueta S, 2015).

En este orden de ideas, es necesario comprender el significado y avance científico que esta herramienta tecnológica aporta a las organizaciones, especialmente en el marketing y los negocios. Cuyo concepto formulado por la American Marketing Association (AMA), lo define como: "la actividad, conjunto de instituciones y procesos para crear, comunicar, entregar e intercambiar ofertas que tienen valor para los consumidores, clientes, socios y la sociedad en general", donde la Big Data empieza a dar valor agregado al generar información después de un procesamiento predeterminado, que logre alcanzar un mejor entendimiento de los gustos, deseos y necesidades del consumidor.

Tal como lo plantean Ortiz M, Joyanes L, Giraldo L, (2016), en una era digital y de continuo cambio, la empresa debe conocer el mercado y las actuaciones de la competencia con el propósito de lanzar productos y servicios. En este sentido se 
requiere que las organizaciones no solo recopilen datos, sino que busquen la forma de analizarlos para concebir actuaciones diarias fundamentadas en estadísticas y tendencias.

Por otra parte, encontramos a Suarez, S. (2014), quien expone que conocer con precisión al consumidor ha sido y es uno de los objetivos más buscados por la publicidad y un punto primordial para la eficiencia en los negocios.

Por lo anterior, este articulo presenta una bibliometría de la relación que existe entre el big data y los negocios no solo en marketig sino al empleo del mismo para una adecuada toma de decisiones en las organizaciones tanto de Colombia, Latinoamérica y el resto del mundo.

\section{MARCO TEÓRICO}

El concepto de Big Data ha evolucionado a ser prioridad empresarial en la era de las Tic, y se caracteriza por sus cuatro dimensiones: Volumen, variedad, velocidad y veracidad. El volumen tiene relación con la cantidad de datos; la variedad se relaciona a la estructura de los datos, la forma en que vienen, ya sea, texto, audio, video, etc; la velocidad está relacionada con el tiempo de accesibilidad a la información o respuesta y la veracidad a la calidad o fiabilidad de los datos (IBM, 2012). Hoy en día el Big Data cobra tanta importancia que incluso se han creado nuevos puestos de trabajo como el denominado Chief Digital Officer (CDO), que se dedica exclusivamente a encontrar información del entorno que pueda ser útil a la organización en los procesos de innovación (Goyzueta, 2015).

Existen diferentes dimensiones del Big Data y la más conocida es el data-driven marketing, o Big Data de Marketing, que consiste en aprovechar la información de los clientes para establecer canales más efectivos de comunicación. Para ello utiliza la recolección y tratamiento de la información de los clientes para alimentar el conocimiento organizacional y fortalecer la fiabilidad hacia la empresa (Goyzueta, 2015). De igual forma brinda al empresario herramientas que le permiten realizar el proceso administrativo como planear, organizar, dirigir y controlar utilizando las TIC en el departamento de marketing (Goyzueta, 2015).
Una oportunidad excepcional del empleo del BD en actividades de marketing en las empresas, es que puede centrar el trabajo sobre el total de datos del universo de estudio, dejando de lado la necesidad de trabajar sobre una muestra (Manovich, 2012). Esta premisa da lugar a un análisis de tendencias mucho más fundamentada, un mayor conocimiento y cobertura del perfil de los clientes que conlleva a actuaciones empresariales con mejores argumentaciones (Goyzueta, 2015) y se convierte en una herramienta que permite involucrar a la estrategia empresarial y de marketing a nuestros clientes y consumidores.

Ahora bien, otro de los elementos del Big Data es la relacionada con los negocios, enfocada a la búsqueda de la sostenibilidad, en el mediano y largo plazo a partir de las relaciones permanentes con los clientes, mayor conocimiento de los procesos, posibilidades de identificar las debilidades y encontrar oportunidades (Camargo-Vega, CamargoOrtega, \& Joyanes-Aguilar, 2015).

De igual forma el Big Data para los negocios, se constituye en una estrategia organizacional para la toma de decisiones en algunas ocasiones en tiempo real en otras con un margen de tiempo mayor, dependiendo de las herramientas y habilidades con las que cuente la organización. Por lo tanto su implementación es una oportunidad para la empresa de estar más cerca del cliente para coinventar soluciones al igual que para convertir las estadísticas en apoyo para la toma de decisiones (Ortiz Morales, Joyanes \& Giraldo, 2015).

Para lograr un impacto importante en los negocios con el uso del Big Data se requiere que los datos estén a disposición de las necesidades de la organización, poder integrar los tipos de datos de variedades diversas, poseer una capacidad para gestionar volúmenes de datos, manejar buenos niveles de seguridad de los datos y realizar planeación y capacitaciones adecuadas al personal (IBM, 2012).

La implementación de una solución Big Data está compuesta de diferentes tecnologías que trabajan conjuntamente para lograr el objetivo final de almacenar y procesar grandes cantidades de datos. Entre las tecnologías involucradas se encuentran el framework del proyecto Apache Hadoop, el uso de base de datos NoSQL entre las que podemos mencionar Cassandra, Hbase, MongoDB, Neo4j, entre otras (Paiva, 2016). 


\section{METODOLOGÍA}

La metodología responde a una investigación cuantitativa de tipo no experimental longitudinal del 2010 al 2017 y utiliza como método el proceso básico de la vigilancia tecnológica. Las fases fueron las siguientes:

Fase 1. Planificación y diseño de la metodología de búsqueda.

Fase 2. Búsqueda en bases de datos. Para identificar el estado de la técnica y de la ciencia, se utilizó las bases de datos de Scopus. La selección de la base de datos Scopus de la editorial Elsevier corresponde a la posibilidad de comparar en el ámbito internacional el tema con el ámbito nacional con una de las bases de datos más utilizadas y con mayor número de artículos. Para la búsqueda de información se utilizó la siguiente ecuación: TITLE-ABS-KEY ( "big data" AND "business" ) AND ( LIMIT-TO ( DOCTYPE , "ar" ) ) AND ( LIMIT-TO ( SUBJAREA, "BUSI" ) )

Fase 3. Procesamiento y análisis de la información. Fase 4. Difusión de resultados.

\section{RESULTADOS Y DISCUSIÓN}

\subsection{Estado de la Ciencia en la relación Big Data y los Negocios}

En la base de datos Scopus, se encontraron 3889 documentos que integran la temática de Big Data, de los cuales 559 se relacionan con negocios. De los resultados se seleccionaron aquellos documentos clasificados como artículos científicos. La búsqueda se redujo a 278 documentos con las siguientes características: Desde el 2010 los científicos inician su interés y publicación de temas sobre Big Data y Negocios con un aumento en publicaciones de 3,2 veces entre el año 2012 al 2014 y de 4.4 veces entre el 2012 y el 2017. Lo que representa interés de los investigadores en el tema que se incrementa a través de los años (Ver Figura 1)

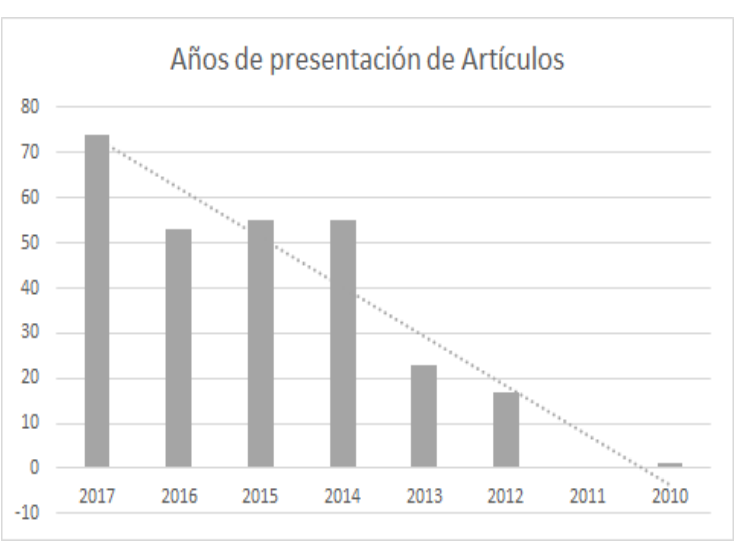

Figura 1. Años de presentación de artículos Fuente: Las autoras a partir de la base de datos Scopus.

Se tomaron las fuentes con más de cinco publicaciones. El mayor número de publicaciones que se hacen sobre Big Data en relación con los negocios la tiene IBM Data Management Magazine, seguido por Industrial Management and Data Systems y el Journal of Business Research. Estas fuentes son revistas en línea que se especializan en la búsqueda de soluciones tecnológicas para la toma de decisiones en las organizaciones (Ver Figura 2).

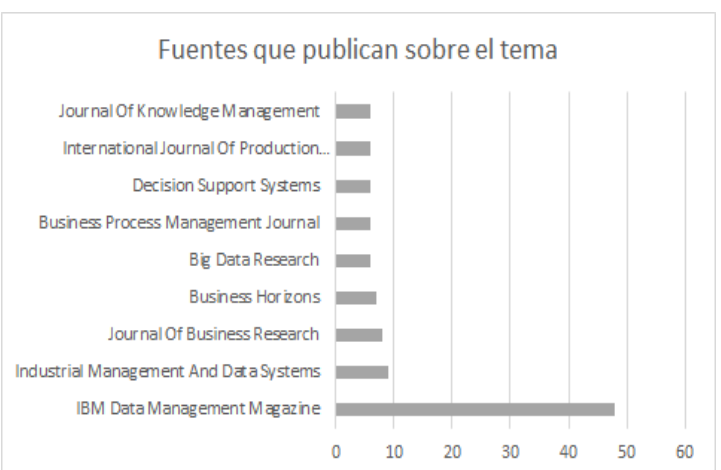

Figura 2. Fuentes que publican sobre el tema Fuente: Las autoras a partir de la base de datos Scopus.

Las publicaciones sobre Big Data tienen diversidad de autores (159). Aplicando la Ley de Lotka, para determinar el índice de productividad se evidencia una mediana especialización de los investigadores en el tema. Los autores con más de 5 artículos sobre Big Data relacionada con los Negocios son: Akter, Shahriar, de la University of Wollongong, Faculty of Business, Wollongong, Australia Fattah, A; Fattah, Ahmed autor en IBM Data Management Magazine donde relaciona el Big Data con los 
negocios y los sistemas. Fosso Wamba, Samuel de NEOMA Business School, Information Systems, Rouen de France (Ver Figura 3) .

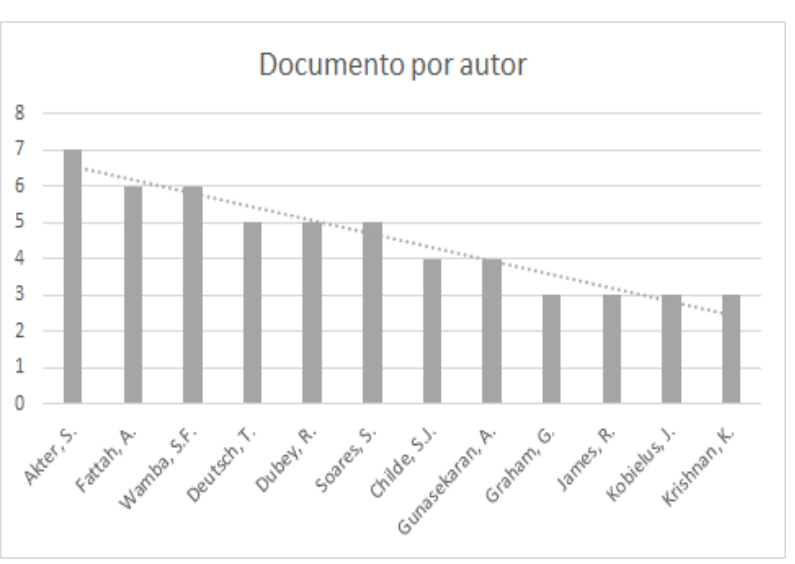

Figura 3. Documentos por autor.

Fuente: Las autoras a partir de la base de datos Scopus.

Las universidades más destacadas por que sus investigadores escriben sobre el tema de Big Data y negocios son: University of Wollongong de Australia, con 7 documentos y Symbiosis International University de India con 6 documentos (Ver Figura 4).

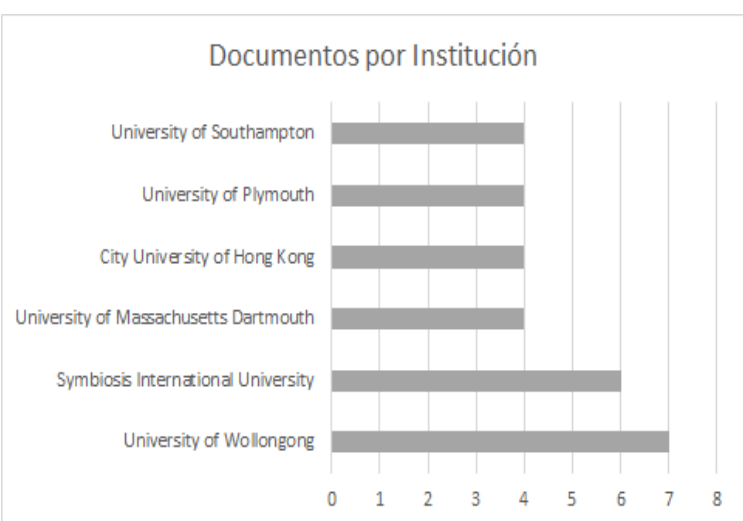

Figura 4. Documentos por institución.

Fuente: Las autoras a partir de la base de datos Scopus.

Los países donde más se escribe sobre el tema son Estados Unido seguido de países como Reino Unido, China, Australia y Alemania (Ver Figura 5).

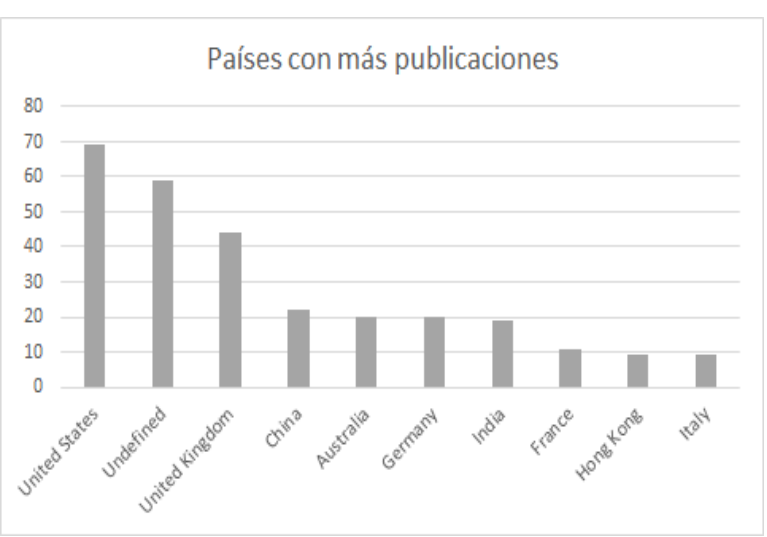

Figura 5. Países con más publicaciones.

Fuente: Las autoras a partir de la base de datos Scopus.

En el ámbito Latinoamericano en la base de datos de Scopus, solo aparecen los siguientes países: Brasil con 6 artículos Chile con 2 y Argentina con 1. En la base de datos de Scopus se evidencian publicaciones sobre el tema a partir del 2010, con una disminución en el 2011 y un aumento progresivo a partir de 2012 (Ver Figura 6).

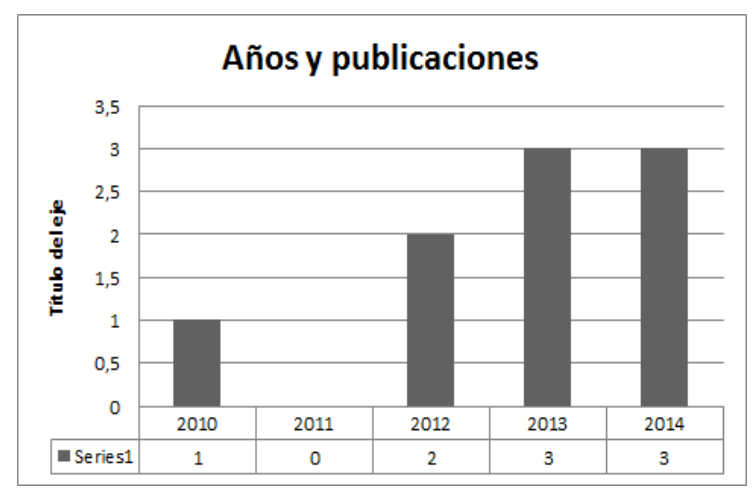

Figura 6. Años y publicaciones.

Fuente: Las autoras a partir de la base de datos Scopus.

La fuente con mayores publicaciones la constituye la revista RAE Revista De Administracao De Empresas, seguida de Mundo Agrario (Ver Figura 7) 


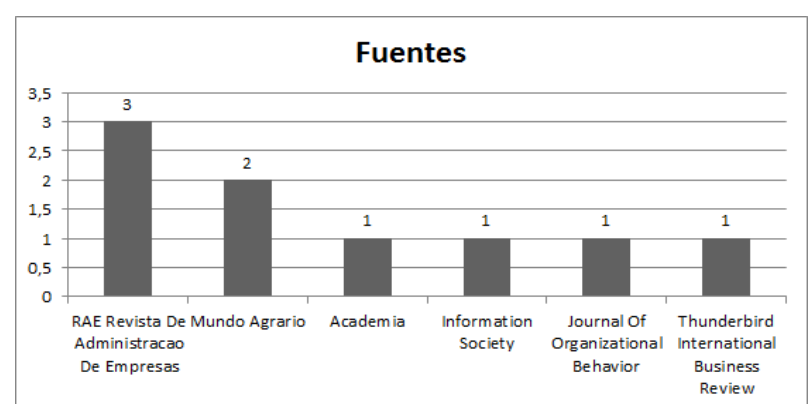

Figura 7. Fuentes.

Fuente: Las autoras a partir de la base de datos Scopus.

\subsection{Estado de la Ciencia en Colombia}

De acuerdo a la plataforma de Colciencias los grupos de investigación han presentado en Colombia dos proyectos relacionados con Big Data y negocios, como se aprecia en la Tabla 1:

Tabla 1. Proyectos de los grupos de investigación en Colombia

\begin{tabular}{lll}
\hline \multicolumn{1}{c}{ Grupo } & \multicolumn{1}{c}{ Proyecto } & Año \\
\hline $\begin{array}{l}\text { CIEN-K (Grupo de } \\
\text { investigación de } \\
\text { estudios }\end{array}$ & $\begin{array}{l}\text { Investigación y } \\
\text { desarrollo: }\end{array}$ & 2015 \\
organizacionales y & $\begin{array}{l}\text { Opportunities and } \\
\text { challenges of using }\end{array}$ & \\
de marketing & $\begin{array}{l}\text { Big Data: The case of } \\
\text { agriculture activities }\end{array}$ & \\
GPC - Gerencia, & $\begin{array}{l}\text { Investigación y } \\
\text { desarrollo: }\end{array}$ & 2015 \\
productividad y & $\begin{array}{l}\text { Framework Big Data } \\
\text { competitividad }\end{array}$ & \\
& $\begin{array}{l}\text { para apoyar el } \\
\text { desarrollo de PYMES } \\
\text { del sector Salud en }\end{array}$ & \\
& Colombia \\
\hline
\end{tabular}

Fuente: Las autoras a partir de la base de datos de Colciencias.

Por otra parte la revisión de información en la plataforma de Colciencias, permitió encontrar 15 productos de grupos de investigación sobre el tema de Big Data relacionados con negocios, como se aprecia en la figura 8 .

Los mayores productos de investigación son los trabajos de pregrado, seguidos de los artículos publicados en revistas especializadas y luego los cursos de corta duración y trabajos de grado de maestría.

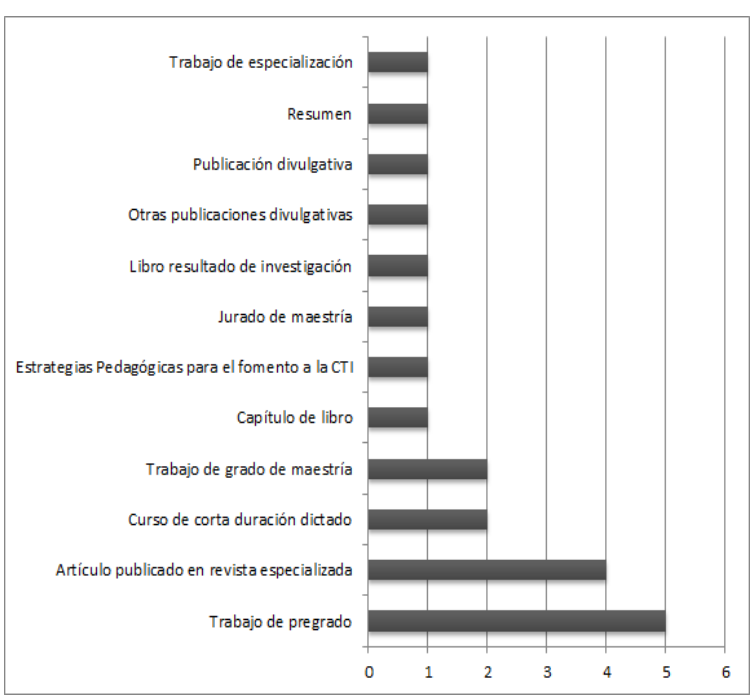

Figura 8. Temas de publicación sobre Big Data y negocios en Colombia.

Fuente: Las autoras a partir de la base de datos de Colciencias.

Los temas que presentan los trabajos de pregrado, como se muestra en la Figura 9, están enfocados en un $40 \%$ al diseño de prototipos mientras otros identifican herramientas, servicios y beneficios del Big Data.

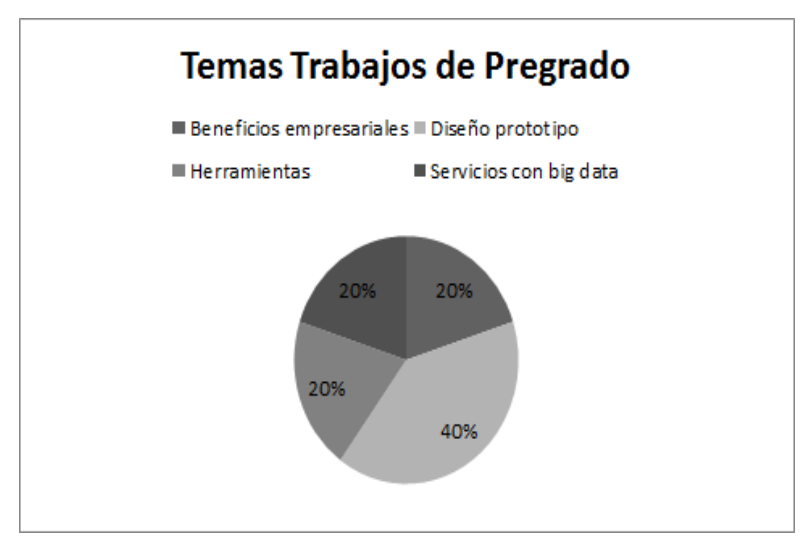

Figura 9. Temas de publicación sobre Big Data y negocios en Colombia.

Fuente: Las autoras a partir de la base de datos de Colciencias.

Ahora bien, los artículos en revistas especializadas que han publicado los grupos de investigación en Colombia centran su atención en aspectos como los usos de Big Data y el cumplimiento de la ética. Las 
acciones del gobierno nacional para fortalecer las tecnologías y el uso y la evolución del Big Data.

Los cursos de corta duración que imparten los grupos de investigación en Colombia son los relacionados con la minería de datos y la innovación en la cultura emprendedora a partir del Big Data, de igual forma los trabajos de grado de maestría que dirigen los investigadores de Colciencias se centran en la minería de datos y la regulación colombiana para el empleo y la optimización del comercio con apoyo del Big Data. El trabajo de especialización dirigido apunta a monitorear la audiencia televisiva empleando Big Data. Mientras que el único capítulo de libro que han publicado los investigadores de Colciencias se enfoca encontrar una relación con el Big Data, la universidad, empresa y Estado.

Por otra parte, el libro The 8th International Conference on Knowledge Management in Organizations, publicado por el grupo de investigación GICOECOL, trata temas sobre gestión de servicios en la nube y bibliometría, que en forma indirecta se relacionan con el Big Data. Por otra parte, las estrategias pedagógicas y las publicaciones divulgativas se centran en aspectos como el control social y la Iberciencia. Se destaca que el grupo de investigación Tecnología de Información ha creado un semillero de investigación, que estudia temas sobre el Big Data y la transferencia de tecnología. Por último, el resumen que el grupo de investigación FICB-PG ha escrito se relaciona con el Big Data y la investigación de marketing.

Una revisión a la plataforma de Publindex para encontrar revistas científicas en Colombia que publiquen sobre el tema de Big data y los negocios permitió encontrar los siguientes resultados (Ver tabla 2)

Tabla 2. Artículos en la base de dato de Publindex relacionados con Big Data y negocios

\begin{tabular}{|c|c|c|c|}
\hline Artículos & Autor (es) & Año & Tema \\
\hline $\begin{array}{l}\text { Tackling the } \\
\text { challenges of } \\
\text { an aging } \\
\text { workforce } \\
\text { with the use } \\
\text { of wearable } \\
\text { technologies }\end{array}$ & $\begin{array}{l}\text { Ferreira } \\
\text { Martins } \\
\text { Arezes,Pedro } \\
\text { Miguel } \\
\text { Lavallière } \\
\text {,Martin - } \\
\text { Burstein }\end{array}$ & 2016 & $\begin{array}{l}\text { Los wearables } \\
\text { pueden } \\
\text { aprovechar y } \\
\text { fortalecer las } \\
\text { intervenciones } \\
\text { para mejorar la } \\
\text { salud, seguridad }\end{array}$ \\
\hline
\end{tabular}

\begin{tabular}{|l|l|l|l|}
\hline $\begin{array}{l}\text { and the } \\
\text { quantified- } \\
\text { self }\end{array}$ & $\begin{array}{l}\text {,Arielle } \\
\text { Coughlin } \\
\text { Joseph - I }\end{array}$ & $\begin{array}{l}\text { y el bienestar de } \\
\text { los empleados, } \\
\text { en especial las } \\
\text { personas de } \\
\text { avanzada edad. }\end{array}$ \\
\hline $\begin{array}{l}\text { Análisis de } \\
\text { la Calidad } \\
\text { del Agua del } \\
\text { Río Bogotá } \\
\text { Durante el } \\
\text { Periodo 2008 } \\
\text {-2015 a } \\
\text { Partir de } \\
\text { Herramientas } \\
\text { de Minería } \\
\text { de Datos }\end{array}$ & S.A. & 2015 & $\begin{array}{l}\text { Analizar y } \\
\text { evaluar el } \\
\text { espacio temporal } \\
\text { de la calidad del } \\
\text { río Bogotá } \\
\text { mediante la } \\
\text { minería de datos. }\end{array}$ \\
\hline
\end{tabular}

Fuente: Las autoras

Al comparar las búsquedas de publicaciones que se hacen sobre Big data como único término de consulta con las que se hacen relacionando el Big data con los negocios, se puede apreciar que Estados Unidos sigue ocupando los primeros lugares, mientras China se desplaza a un tercer lugar. Así también el número de documentos se hace mucho menor, existiendo 5.393 publicaciones en Estados Unidos sobre esta temática. Mientras que relacionado con los negocios solo aparecen 69 publicaciones. En el caso Latinoamericano sigue siendo Brasil el líder en publicaciones reportadas en Scopus sobre Big data, con 304 publicaciones, en Colombia aparecen (23) publicaciones, superada por México (74) y Chile (46) y al mismo nivel de Argentina (23) (Secretaría de Planeamiento y Políticas del Ministerio de Ciencia, Tecnología e Innovación Productiva de la República Argentina, 2015).

La estrategia de Big Data en los negocios no está solamente en su implementación pues se hace necesario no solo capturar la información sino sacarle provecho para mejorar la competitividad. Por lo tanto, poseer la información no es el fin del proceso, las empresas deben aprender y encontrar las mejoras prácticas para pasar al plano en el que las acciones empiezan a tomar un rol decisivo. Se pueden presentar obstáculos de carácter técnico como una inadecuada arquitectura establecida, con demasiados nodos o con muy pocos puntos de integración, o también obstáculos de tipo cultural como decisiones mal implementadas en las estructuras jerárquicas, baja confianza en los nuevos modelos o simplemente que las áreas del negocio se muestren reacias a compartir la 
información entre ellas. En la mayoría de las situaciones los problemas surgen de una mezcla de estos factores (Rodríguez, 2016).

El porcentaje de proyectos de Big Data en Colombia puestos en producción durante el 2014, es del 13\%. Las organizaciones han trabajado en la recolección de datos, la realización de pilotos y otros experimentos, acciones que reflejan una evolución y mucha voluntad para explorar y aprovecharse de las grandes oportunidades que puede ofrecer el análisis de datos. Es notable el aumento de las compañías, de todos los sectores e industrias, que están invirtiendo en proyectos de Big data. La adopción de proyectos Big data es un reto no sólo del sector privado, sino de las instituciones públicas que están cada vez más comprometidas (Maroto, 2015).

\section{CONCLUSIONES}

El Big Data se constituye en una estrategia para fortalecer el manejo adecuado de las grandes bases de datos y poder convertir los datos en información y conocimiento al servicio de las organizaciones no solo en marketing sino en un plano más estratégico de la organización.

En comparación con Latino américa y el resto del mundo Colombia se encuentra rezagada en publicaciones científicos que relacionen el Big Data con los negocios y las pocas que existen hacen énfasis en el diseño de prototipos y beneficios del Big Data.

Se recomienda fortalecer investigaciones aplicadas sobre Big Data en relación a los negocios para implementar los beneficios y sacarle provecho que redunde en la mejora de la competitividad de las organizaciones.

\section{REFERENCIAS}

American Marketing Association. (2015). Marketing Dictionary. Recuperado de https://www.ama.org/resources/Pages/Dictionary.as px?dLetter=M

Camargo-Vega, Juan José, Camargo-Ortega, Jonathan Felipe, \& Joyanes-Aguilar, Luis. (2015). Conociendo Big Data. Facultad de Ingeniería, 24(38), 63-77.
Carlos Parra Ortega, Jaime Herrera Vergara. (2013). Aplicación de los sistemas de detección de intrusos y la tecnología de agentes en el monitoreo inteligente de redes de datos. Revista Colombiana de Tecnologías de Avanzada ISSN: 1692-7257 - Volumen 2 - Número 22.

Drucker, P. F. (1973). Management: tasks, responsibilities, practices. Nueva York: Harper and Row Publishers.

Giovanny Sandoval, Jacobo Tobar Molano, Víctor Hugo Mosquera, Luis Jorge González (2011). Pluviógrafo electrónico con transmisión de datos inalámbrica. Revista Colombiana de Tecnologías de Avanzada ISSN: 1692-7257 - Volumen 1 Número 17.

Goyzueta, R. (2015). Big Data Marketing: una aproximación. Revista Perspectivas, (35), 147-158.

IBM (2012). Analytics: El uso del Big Data en el mundo real. Cómo las empresas más innovadoras extraen valor de datos inciertos España: IBM.

Recuperado de: https://www05.ibm.com/services/es/gbs/consulting/pdf/El_uso_ de_Big_Data_en_el_mundo_real.pd

Jaime Guzmán-Luna, Ingrid-Durley Torres, Juan Felipe Alvarez (2014). Propuesta de un generador de aplicaciones educativas basadas en televisión digital usando arquitectura de cómputo en la nube. Revista Colombiana de Tecnologías de Avanzada ISSN: 1692-7257 - Volumen 2 Número 24.

Luis A Mesa Mesa, Nelson Barrera Lombana (2013). La robótica educativa como instrumento didáctico alternativo en educación básica. Revista Colombiana de Tecnologías de Avanzada ISSN: 1692-7257 - Volumen 2 - Número 22.

Maroto, C (2015). Big Date aquí y ahora 2015: Situación mundial $\mathrm{y}$ foco en el mercado de Colombia. Colombia: Business Scholl

María Claudia Bonfante, Andrés Castillo (2014). Integración de sistema multi-agente, ontologías y procesos de negocios como marco tecnológico de la estrategia "gobierno en línea". Revista Colombiana de Tecnologías de Avanzada ISSN: 1692-7257 Volumen 1 - Número 23.

Manovich, L. (2012).'Trending: the promises and the challenges of Big Social Data', en Gold, M. G. (2012). Debates in the digital humanities. Arizona: University of Minessota Press.

Malvicino, F. y Yoguel, G. (2014), Big Data. Avances Recientes a Nivel Internacional y Perspectivas para el Desarrollo Local. Documento de Trabajo. Centro Interdisciplinario de Estudios en Ciencia Tecnología e Innovación (CIECTI - MinCyT) 
Ortiz Morales, M., Joyanes Aguilar, L., \& Giraldo Marín, L. (2015). Los desafíos del marketing en la era del big data. e-Ciencias de la Información. , 6(1), 1-31

Ortiz M, Joyanes L, Giraldo L, (2016), Los desafíos del marketing en la era del big data. e-Ciencias de la Información, 6 (1), recuperado de DOI: http://dx.doi.org/10.15517/eci.v6i1.19005

Paiva, P. (2016). Desarrollo de una Arquitectura Big Data para Registros Mercantiles. (Tesis de pregrado). Universidad central, Venezuela.

Paniagua E, (2014). Big data el poder de los datos. Fundación Innovación Bankinter. Recuperado de www.fundacionbankinter.org

Rodriguez, J. (2016). Implementación de Big data en las organizaciones como estrategia de aprovechamiento de la información para incorporar a la cadena de valor del negocio (tesis de posgrado).Universidad Militar Nueva Granada, Bogotá, Colombia.

Russom, P. (2012). Big Data Analytics, TDWI (TheData Warehousing Institute).

Secretaría de Planeamiento y Políticas del Ministerio de Ciencia, Tecnología e Innovación Productiva de la República Argentina. (2015). Medición de la producción científica en Big Data. Recuperado de: http://indicadorescti.mincyt.gob.ar/documentos/Me dicion_produccion_cientifica_big_data.pdf

Suarez S, (2014). Desafíos y oportunidades de la analítica big data aplicada a la actividad publicitaria. Disponible en https://repositori.upf.edu/bitstream/handle/10230/2 5263/Suarez_2015.pdf?sequence $=1$

Tang Tong M, (2015). La inteligencia de mercado en las empresas exportadoras e importadoras peruanas. Ingeniería Industrial, 33, 71-97 